\title{
IN VITRO ANTIMYCOTIC EVALUATION AND PRELIMINARY COMBINATORIAL STUDIES OF IBUPROFEN WITH STANDARD ANTIFUNGAL DRUGS AGAINST ASPERGILLUS SPP.
}

\author{
MRRIDULA DANGI NARWAL, MEENAKSHI BALHARA, RENU CHAUDHARY, ANIL KUMAR CHHILLAR*
}

Centre for Biotechnology, Maharshi Dayanand University, Rohtak, Haryanan, India. Email: anil.chhillar@gmail.com

Received: 31 March 2017, Revised and Accepted: 21 April 2017

\section{ABSTRACT}

Objective: The prevalence of invasive mycoses is increased in the immunocompromised patients with an increase in resistance developed against current antifungal drugs. This has led to the need for discovering novel combinations of the antifungal drugs to combat against resistant pathogenic spp. This study mainly targets to evaluate the antifungal activity of ibuprofen (IBU) alone and in combination with the standard antifungal drugs (polyenes and azoles) against eight isolates of Aspergillus fumigatus, Aspergillus flavus, and Aspergillus niger.

Methods: The study was performed using the disc diffusion assay (DDA), microbroth dilution assay and spore germination inhibition assay. Moreover, cytotoxicity was checked by heamolytic assay.

Results: Minimum inhibitory concentration (MIC) of IBU against A. fumigatus and A. flavus using DDA is found to be in the range of $250-275 \mu \mathrm{g} / \mathrm{disc}$ while for $A$. niger isolates, the range was 500-575 $\mu \mathrm{g}$ /disc. Likewise, by broth microdilution assay and spore germination inhibitory assay, MIC determined, were in the range of 500-750 $\mathrm{gg} / \mathrm{ml}$ against $A$. fumigatus and A. flavus while for A. niger, it was $1000-1500 \mu \mathrm{g} / \mathrm{ml}$.

Conclusion: IBU demonstrated its antimycotic potential against all the eight isolates of Aspergillus spp. Moreover, preliminary combinatorial evaluation of IBU with the standard antifungal drugs reported by DDA revealed an increase in zone of inhibition as compared to the drugs alone. Further research regarding the confirmation of synergistic interaction between the selected drugs is in progress.

Keywords: Aspergillosis, Antifungal, Combination, Ibuprofen.

(C) 2017 The Authors. Published by Innovare Academic Sciences Pvt Ltd. This is an open access article under the CC BY license (http://creativecommons. org/licenses/by/4. 0/) DOI: http://dx.doi.org/10.22159/ajpcr.2017.v10i8.18188

\section{INTRODUCTION}

A prevalence of the opportunistic mycotic infections has varied for the past few decades. Invasive aspergillosis (IA), predominantly caused by Aspergillus fumigatus, followed by Aspergillus flavus and Aspergillus niger, have increased the risk of morbidity and mortality among immunosuppressed cases [1,2]. Although, for the past few decades, antimycotic agents such as polyenes, azoles, and echinocandins have proved to act significantly against IA $[3,4]$. However, the resistances against these standard antimycotic agents have developed the urge to search for the new drugs that can help eradicate severe opportunistic mycotic infections like IA. Moreover, lack of the effective monotherapy and the combination therapy of the standard drugs against IA has further allowed the researchers to develop new combinations that can lead to better therapeutic treatment for IA.

Ibuprofen (IBU) is a derivative of the propionic acid and relates to the class of non-steroidal anti-inflammatory drugs (NSAID), known for its anti-inflammatory, analgesic and anti-pyretic properties [5]. IBU inhibits the synthesis of prostaglandins from the arachidonic acid as it has the capability to obstruct the cyclo-oxygenase enzyme $[5,6]$. Earlier studies have demonstrated the antifungal activity of IBU and its synergy with the azoles against Candida spp. [7,8]. In addition, the IBU has also been reported to induce blocking of the efflux pumps such that reverting resistant Candida spp. into susceptible ones, against azoles $[9,10]$.

Thus, in this study, antifungal activity of IBU has been evaluated alone and in combination with standard antifungal drugs, the polyenes: Amphotericin B (AmpB), nystatin (NYST) and the azoles: Ketoconazole
(KETZ), flucanozole (FLUZ), via., various techniques against three clinical isolates of $A$. fumigatus, three of $A$. flavus and two clinical strains of $A$. niger.

\section{METHODS}

The antifungal drugs, AmpB, NYST, KETZ, and FLUZ obtained from Himedia Chemicals and IBU from the Fluka Analytical Chemicals. $4 \mathrm{mg} / \mathrm{ml} \mathrm{AmpB}$ and $10 \mathrm{mg} / \mathrm{ml}$ of IBU were taken as the stock solution and diluted with the distilled water to get the required concentration depending on the experiment done.

\section{Pathogens}

Pathogenic strains of A. fumigatus (clinical isolate 190/96 [Vallabhbhai Patel Chest Institute, Delhi (VPCI) and ITCC1634 [Indian Agricultural Research Institute Delhi (IARI)] and ITCC 4517 [IARI, Delhi]), A. flavus (clinical isolate 223/96 [VPCI, Delhi], ITCC 5192 [IARI, Delhi], and ITCC 5076 [IARI, Delhi]), and A. niger (clinical isolate 56/96 [VPCI, Delhi] and ITCC 5405 [IARI, Delhi]) were used in the current research. All the isolates were grown on sabouraud dextrose (SD) agar culture Petri plates of $10.0 \mathrm{~cm}$ diameter (tarsons) by inoculating with stock of A. fumigatus, A. flavus, and A. niger and incubating at $37^{\circ} \mathrm{C}$ in a biochemical-oxygen demand incubator. After $96 \mathrm{hrs}$ incubation, spores were collected from culture plates and distributed evenly in phosphate buffer saline (PBS). The spores were collected and a concentration of $10^{8}$ spores/mL was used to perform the experiments.

\section{Assay}

The antimycotic activity of IBU was analyzed by the disc diffusion assay (DDA), microbroth dilution assay (MDA), and spore germination inhibition assays (SGIA) as illustrated previously $[4,11]$. 
DDA

DDA was performed using the radiation sterilized Petri plates cultured with the SD agar and inoculated with $100 \mu \mathrm{l}$ of homogenized suspension of $1 \times 10^{8}$ spores $/ \mathrm{ml}$ of Aspergillus spp. Discs of $5.0 \mathrm{~mm}$ in diameter (Whatman filter paper No. 1) were placed on dried plates. Furthermore, discs were impregnated with the different concentrations of IBU in the range of 125-1000 $\mu \mathrm{g} /$ disc and AmpB within range of 5-0.35 $\mu \mathrm{g} / \mathrm{disc}$. After incubation at $37^{\circ} \mathrm{C}$, Petri plates were observed at 24 and 48 hrs to check if there was any zone of inhibition, around the discs to know the antifungal effect.

\section{MDA}

The assay was executed in 96-well microtiter flat bottom plates (Tarson). Different dilutions of IBU starting with $1000 \mu \mathrm{g} / \mathrm{ml}$ were prepared in $100 \mu \mathrm{l}$ of culture medium. Moreover, wells were administered with $10 \mu \mathrm{l}$ of spores $\left(1 \times 10^{8}\right.$ spores $\left./ \mathrm{ml}\right)$. 96 -well plates were then incubated at $37^{\circ} \mathrm{C}$ and observed visually after $48 \mathrm{hrs}$. The well with no growth, i.e., complete inhibition of growth, was taken as end point and considered minimum inhibitory concentration (MIC).

\section{SGIA}

Different concentrations of IBU in $100 \mu \mathrm{l}$ of culture medium were prepared in 96-well plates by double dilution method. Inoculation of each well was done with $10.0 \mu \mathrm{l}$ of spore suspension containing $100 \pm$ 5 spores. After $16 \mathrm{hrs}$ of incubation at $37^{\circ} \mathrm{C}$, microtitre plates were assessed for germination of spores with an inverted microscope. The number of germinated and non-germinated spores will be counted. The percentage of inhibition of spore germination was calculated by the formula described by Ruhil et. al. [4]. The least concentration of the IBU, which was responsible for more than $90 \%$ inhibition of spore germination was taken as MIC $_{90}$.

\section{Toxicity studies}

\section{Haemolytic assay}

This method $[4,11]$ was performed using the erythrocytes from the healthy humans was taken and rinsed them with autoclaved PBS. Erythrocyte suspension $(2.0 \% \mathrm{v} / \mathrm{v})$ was prepared and exposed with IBU and $\mathrm{AmpB}$ starting with concentration of $1000 \mu \mathrm{g} / \mathrm{ml}$ at $37^{\circ} \mathrm{C}$ for $1 \mathrm{hr}$. The eppendorph tubes were allowed to centrifuge at $5000 \mathrm{rpm}$ (rotation per minute) for 10 minutes. Optical density of the supernatant was calculated at $415 \mathrm{~nm}$ by spectrophotometer (Ultraviolet-visible Spect Lambda Bio 20 Perkin-Elmer). Hemolysis done by the test compounds were measured in percentage and the concentration responsible for $<10 \%$ hemolysis was considered non-toxic. PBS was used as the negative controls while triton $\mathrm{X}$ as positive controls along with the erythrocytes.

\section{In vitro combinatorial evaluation}

In vitro combinatorial evaluation (preliminary) among polyenes and azoles with NSAID IBU was performed using DDA. The $6 \mathrm{~mm}$ discs of Whatmann filter paper one were inoculated with $1.1 \mu \mathrm{g} / \mathrm{disc}$ of AmpB, NYST, KETZ, FLUZ, and MIC of KETZ and FLUZ, as calculated by DDA) alone and with $129 \mu \mathrm{g}$ of IBU. Incubating the plates at $37^{\circ} \mathrm{C}$ for $48 \mathrm{hrs}$ allowed the zone of inhibition to be visibly clear such that radius was measured after an interval of 24 and $48 \mathrm{hrs}$. No antifungal potential was declared where zone of inhibition was below $6 \mathrm{~mm}$.

\section{RESULTS AND DISCUSSION}

In vitro antimycotic potential

The MIC of AmpB, KETZ, FLUZ, NYST by using DDA was $0.76,6.24,31.26$, and $1.1 \mu \mathrm{g} / \mathrm{disc}$, respectively, against all the eight pathogenic stains of Aspergillus genera. The mean of diamtere of zone of inhibition along with standard deviation was also measured for IBU as shown in Table 1.

Remarkably, IBU created an optically clear zone of inhibition of mycotic growth against all eight Aspergillus isolates. The geometric mean (GM) MIC of AmpB (standard antifungal), against three isolates of A. fumigatus, A. flavus and two of A. niger was $1.6 \mu \mathrm{g} / \mathrm{ml}$, by MDA and SGIA. The GM of MIC of IBU has been summarized in Table 2 .
The antifungal activity of IBU against the most pathogenic strain, i.e., A. fumigatus (VPCI190/96) was clearly noticeable with DDA, MDA, SGIA shown in Figs. 1-3. These outcomes established that IBU have natural activity against the Aspergillus spp.

The fungal isolates were observed to show disparity in their susceptibility when they were exposed with the IBU in different culture media. The culture media can influence the intensity of antimycotic activity [12]. Consequently, in vitro activity of the IBU against all the eight isolates of Apergillus spp were examined in three test media, i.e., RPMI 1640, potato dextrose broth (PD broth) and SD broth, concluded in Table 3.

\section{Toxicity test}

Complete lysis of erythrocytes is done by AmpB, NYST, KETZ at a concentration of $37.30,67.2$, and $124 \mu \mathrm{g} / \mathrm{ml}$, respectively. However, IBU was non toxic $>1 \mathrm{mg} / \mathrm{ml}$ (Fig. 4).

\section{Combination study}

The polyenes (AmpB and NYST), azoles (KETZ and FLUZ) alone and with IBU was infused on the discs; data have been summarized in Table 4 after calculating zone of inhibition.

The combination of IBU with FLUZ displayed no activity almost or very less potential while increased zone of inhibition reported maximum in a combination of IBU with polyenes and also has synergy with KETZ.

Table 1: MIC determination of IBU by DDA

\begin{tabular}{|c|c|c|c|}
\hline \multirow[t]{2}{*}{ Drugs } & \multicolumn{3}{|c|}{ Mean of diameter of zone of inhibition } \\
\hline & \multicolumn{3}{|c|}{ Pathogens Mean \pm SD $(\mathrm{mm})$} \\
\hline NSAID & A.fumigatus (3) ${ }^{\#}$ & A. niger (2) \# & A. flavus $(3)^{\#}$ \\
\hline \multicolumn{4}{|c|}{ IBU $(\mu \mathrm{g} /$ disc $)$} \\
\hline 1000 & $11.5 \pm 0.1$ & $10.4 \pm 0.2$ & $11.3 \pm 0.2$ \\
\hline 500 & $8.9 \pm 0.2$ & $7.3 \pm 0.3$ & $8.8 \pm 0.1$ \\
\hline 250 & $6.8 \pm 0.1$ & NAP & $6.7 \pm 0.2$ \\
\hline 125 & NAP & NAP & NAP \\
\hline \multicolumn{4}{|l|}{ AmpB } \\
\hline 0.76 & $6.4 \pm 0.2$ & $6.5 \pm 0.2$ & $6.4 \pm 0.1$ \\
\hline
\end{tabular}

SD: Standard deivaiton, NAP: No antifungal potential, "Number of strains, NSAID: Non-steroidal anti-inflammatory drugs, IBU: Ibuprofen, MIC:, DDA: Disc diffusion assay, A. fumigatus: Aspergillus fumigatus, A. flavus: Aspergillus flavus, A. niger: Aspergillus niger, AmpB: Amphotericin B

Table 2: Summary of GM of MIC of IBU by different assays

\begin{tabular}{llll}
\hline Pathogens & \multicolumn{2}{l}{ GM*MIC of IBU } & \\
\cline { 2 - 4 } & MDA $(\boldsymbol{\mu g} / \mathbf{m l})$ & DDA $(\boldsymbol{\mu g} / \mathbf{m l})$ & SGIA $(\boldsymbol{\mu g} / \mathbf{m l})$ \\
\hline A. fumigatus (3) & 572.35 & 258.07 & 572.35 \\
A. niger (2) & 1224.74 & 536.19 & 1224.74 \\
A. flavus (3) & 572.35 & 258.07 & 572.35 \\
\hline
\end{tabular}

*GM: Geomatric mean, MIC: Minimum inhibitory concentration, IBU: Ibuprofen, MDA: Microbroth dilution assay, DDA: Disc diffusion assay, SGIA: Spore germination inhibition assays, A. fumigatus: Aspergillus fumigatus, A. flavus: Aspergillus flavus, A. niger: Aspergillus niger

Table 3: In vitro activity of IBU in different test media

\begin{tabular}{llll}
\hline Pathogens & \multicolumn{2}{l}{ GM MIC of IBU } & \\
\cline { 2 - 4 } & SD broth & PD broth & RPMI 1640 \\
\hline A. fumigatus (3) & 572.35 & 314.980 & 721.12 \\
A. niger (2) & 1224.74 & 1000 & 1732.05 \\
A. flavus (3) & 572.35 & 314.980 & 721.12 \\
\hline
\end{tabular}

IBU: Ibuprofen, GM: Geometric mean, MIC: Minimum inhibitory

concentration, A. fumigatus: Aspergillus fumigatus, A. flavus: Aspergillus flavus,

A. niger: Aspergillus niger, PD broth: Potato dextrose broth 
Table 4: In vitro combination of the standard drugs with IBU against the Aspergillus spp. by DDA

\begin{tabular}{|c|c|c|c|c|c|c|}
\hline \multirow[t]{4}{*}{ Drugs $(\mu \mathrm{g} / \mathrm{disc})$} & \multicolumn{6}{|c|}{ Mean diameter zone of inhibition (mm) } \\
\hline & \multicolumn{6}{|c|}{ Pathogens } \\
\hline & \multicolumn{2}{|c|}{ A. fumigatus (3) } & \multicolumn{2}{|c|}{ A. niger (2) } & \multicolumn{2}{|c|}{ A. flavus (3) } \\
\hline & 24 hrs & 48 hrs & 24 hrs & 48 hrs & 24 hrs & 48 hrs \\
\hline AmpB (1.1) & $7.3 \pm 0.1$ & $7.2 \pm 0.2$ & $6.8 \pm 0.2$ & $6.5 \pm 0.1$ & $7.3 \pm 0.1$ & $7.2 \pm 0.2$ \\
\hline NYST (1.1) & $6.9 \pm 0.1$ & $6.4 \pm 0.2$ & $6.5 \pm 0.2$ & $6.1 \pm 0.1$ & $6.9 \pm 0.1$ & $6.4 \pm 0.2$ \\
\hline FLUZ (31.26) & $6.2 \pm 0.2$ & $6.0 \pm 0.2$ & $6.1 \pm 0.1$ & NAP & $6.2 \pm 0.2$ & $6.0 \pm 0.2$ \\
\hline IBU (129) & NAP & NAP & NAP & NAP & NAP & NAP \\
\hline AmpB:IBU (1.1:129) & $12.8 \pm 0.1$ & $12.6 \pm 0.2$ & $12.0 \pm 0.1$ & $11.8 \pm 0.2$ & $12.8 \pm 0.1$ & $12.6 \pm 0.2$ \\
\hline NYST:IBU (1.1:129) & $7.8 \pm 0.1$ & $7.6 \pm 0.2$ & $7.3 \pm 0.17$ & $2 \pm 0.17$ & $8 \pm 0.1$ & $7.6 \pm 0.2$ \\
\hline KETZ:IBU (6.24:129) & $7.0 \pm 0.2$ & $6.8 \pm 0.3$ & $6.9 \pm 0.1$ & $7.1 \pm 0.1$ & $7.0 \pm 0.2$ & $6.8 \pm 0.3$ \\
\hline FLUZ:IBU (31.26:129) & $6.5 \pm 0.2$ & $6.4 \pm 0.3$ & $6.3 \pm 0.2$ & $6.2 \pm 0.2$ & $6.5 \pm 0.2$ & $6.4 \pm 0.3$ \\
\hline
\end{tabular}

NAP: No antifungal potential, A. fumigatus: Aspergillus fumigatus, A. flavus: Aspergillus flavus, A. niger: Aspergillus niger, IBU: Ibuprofen, AmpB: Amphotericin B, NYST: Nystatin, KETZ: Ketoconazole, FLUZ: Flucanozole, DDA: Disc diffusion assay

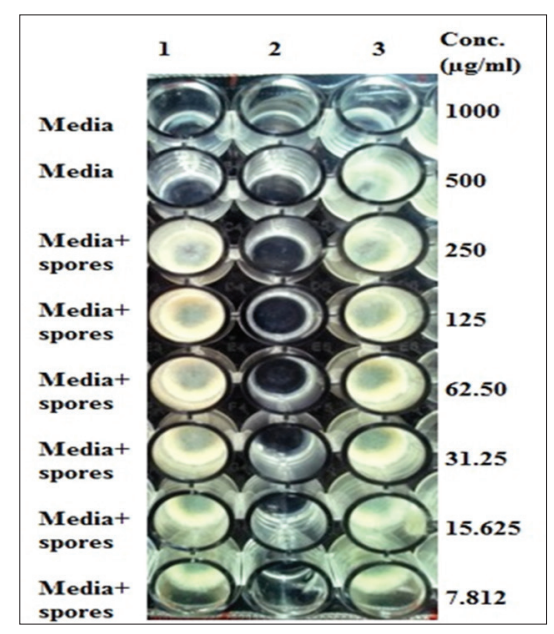

Fig. 1: Microbroth dilution assay of ibuprofen against Aspergillus fumigatus (Vallabhbhai Patel Chest Institute, 190/96)

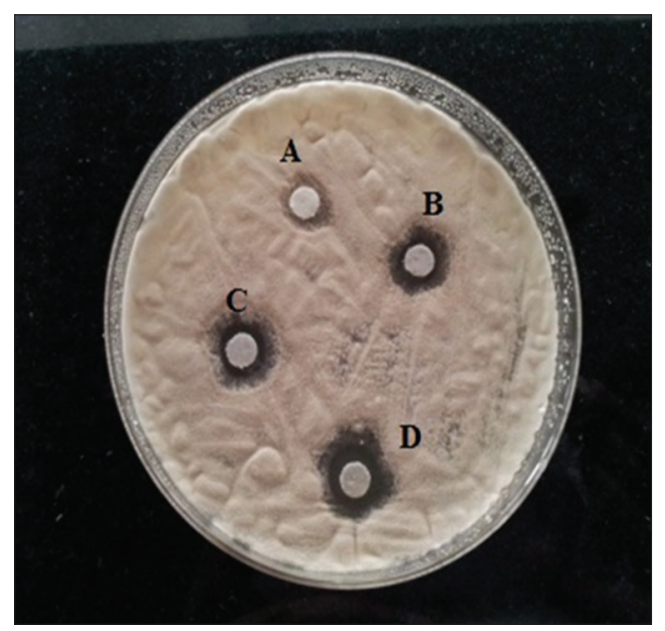

Fig. 2: Disc diffusion assay of non-steroidal anti-inflammatory drugs (ibuprofen) against Aspergillus fumigatus (Vallabhbhai

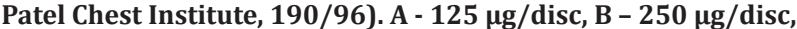
$\mathrm{C}-\mathbf{5 0 0} \mu \mathrm{g} / \mathrm{disc}, \mathrm{D}-\mathbf{1 0 0 0} \mu \mathrm{g} / \mathrm{disc}$

These data suggest that IBU have positive interactions with polyenes and KETZ

Our results clearly portrayed that NSAID can indisputably be administered as antimycotic agent to boost efficiency of current

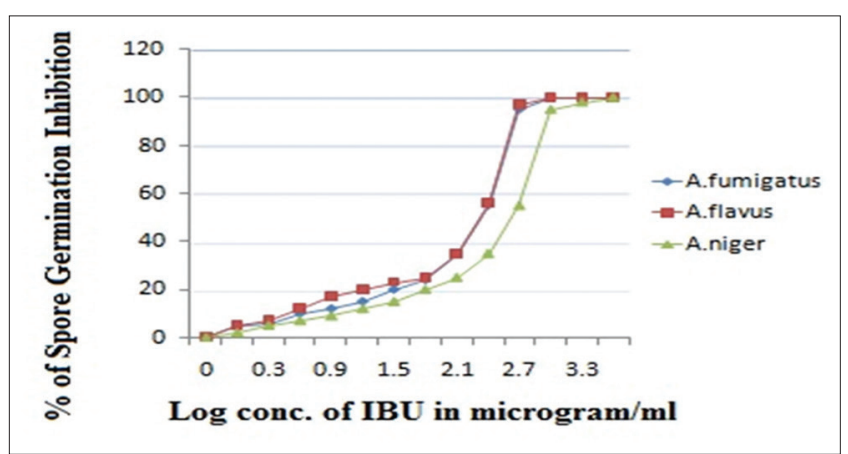

Fig. 3: Spore germination inhibition assays of ibuprofen against Aspergillus spp.

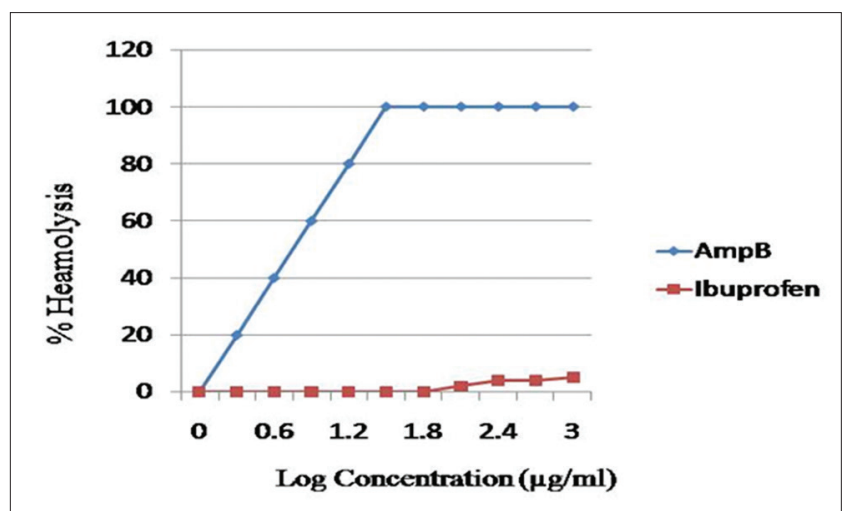

Fig. 4: Toxicity test of ibuprofen by heamolytic assay

antimycotic drugs as IBU is non-toxic even at higher concentrations than standard antifungals so there cytotoxicity might get reduced while administered in combination.

\section{CONCLUSION}

Constant investigations for proficient prevention of IA have been in progress for the past few decades. As a result, alternative drugs whose antifungal properties are still unknown can be tested against pathogenic opportunistic mycotic agents such as IBU. Their interaction with the standard antifungal agents (polyenes and azoles) reduced the toxic effect associated with the current drug monotherapies while increasing their antimycotic activity. Our study displayed synergistic and effective interaction between NSAID IBU and polyenes (maximum). Further research regarding confirmation of synergistic interactions between the selected drugs is in progress. 


\section{ACKNOWLEDGMENTS}

Mrridula et al. would like to thank Centre for Biotechnology, Maharshi Dayanand University, Rohtak, India, Rohtak for the necessary financial support.

\section{REFERENCES}

1. Steinbach WJ, Marr KA, Anaissie EJ, Azie N, Quan SP, Meier-Kriesche HU, et al. Clinical epidemiology of 960 patients with invasive aspergillosis from the PATH Alliance registry. J Infect 2012;65(5):453-64.

2. Naaraayan A, Kavian R, Lederman J, Basak P, Jesmajian S. Invasive pulmonary aspergillosis - Case report and review of literature. J Community Hosp Intern Med Perspect 2015;5(1):26322.

3. Nandhini SU, Bharathy PJ, Rekha S. Antifungal compounds from marine Streptomyces. Int J Pharm Pharm Sci 2015;7(1):207-9.

4. Ruhil S, Balhara M, Dhankhar S, Kumar M, Kumar V, Chhillar AK. Advancement in infection control of opportunistic pathogen (Aspergillus Spp.): Adjunctive agents. Curr Pharm Biotechnol 2013;14(2):226-32.

5. Li-Juan L, Wei C, Hui X, Zhe W, Ruo-yu L, Wei L. Antifungal activity of ibuprofen against Aspergillus species and its interaction with common antifungal drugs. Chin Med J 2010;123 Suppl 19:2701-5.

6. Hersh EV, Moore PA, Ross GL. Over-the-counter analgesics and antipyretics: A critical assessment. Clin Ther 2000;22(5):500-48.

7. Costa-de-Oliveira S, Miranda IM, Silva-Dias A, Silva AP, Rodrigues AG, Pina-Vaz C. Ibuprofen potentiates the in vivo antifungal activity of fluconazole against Candida albicans murine infection. Antimicrob Agents Chemother 2015;59(7):4289-92

8. Venturini TP, Rossato L, Spader TB, Tronco-Alves GR, Azevedo MI, Weiler CB, et al. In vitro synergisms obtained by amphotericin B and voriconazole associated with non-antifungal agents against Fusarium Spp. Diagn Microbiol Infect Dis 2011;71(2):126-30.

9. Medeiros CI, Silva DD, Filho GG, Filho AA, Lima EE. Activity anti-c. Tropicalis and effects of the combination of (s)-(-)-citronellal with four antifungal applied in vulvovaginal candidiasis. Int J Pharm Pharm Sci 2016;8(8):347-51.

10. Ricardo E, Costa-de-Oliveira S, Dias AS, Guerra J, Rodrigues AG, Pina-Vaz C. Ibuprofen reverts antifungal resistance on Candida albicans showing overexpression of CDR genes. FEMS Yeast Res 2009;9(4):618-25.

11. Chhillar AK, Arya P, Mukherjee C, Kumar P, Yadav Y, Sharma AK, et al. Microwave-assisted synthesis of antimicrobial dihydropyridines and tetrahydropyrimidin-2-ones: Novel compounds against aspergillosis. Bioorg Med Chem 2006;14(4):973-81.

12. Rex JH, Cooper CR Jr, Merz WG, Galgiani JN, Anaissie EJ. Detection of amphotericin B-resistant Candida isolates in a broth-based system. Antimicrob Agents Chemother 1995;39(4):906-9. 